University of Wollongong

Research Online

Faculty of Engineering - Papers (Archive)

Faculty of Engineering and Information

Sciences

July 2000

\title{
Characterisation of indium phosphide using terahertz radiation
}

R. L. Causley

University of Wollongong

R. A. Lewis

University of Wollongong, roger@uow.edu.au

Follow this and additional works at: https://ro.uow.edu.au/engpapers

Part of the Engineering Commons

https://ro.uow.edu.au/engpapers/61

\section{Recommended Citation}

Causley, R. L. and Lewis, R. A.: Characterisation of indium phosphide using terahertz radiation 2000.

https://ro.uow.edu.au/engpapers/61

Research Online is the open access institutional repository for the University of Wollongong. For further information contact the UOW Library: research-pubs@uow.edu.au 
Characterisation of indium phosphide using terahertz radiation

\author{
R. L. Causley and R. A. Lewis
}

Institute for Superconducting and Electronic Materials, University of Wollongong, Wollongong NSW 2522, Australia

\begin{abstract}
Specimens of undoped, $n$-type and $p$-type bulk InP have been investigated using $\mathrm{THz}$ radiation. Reflection spectroscopy permits determination of the TO and LO phonon energies as well as the carrier concentration. Absorption spectroscopy reveals transitions between impurity states. We report the first absorption spectrum of an acceptor in InP. The transition energies agree well with electronic Raman scattering and photoluminescence data.
\end{abstract}

\title{
A. Introduction
}

The III-V alloy InP finds considerable technological application. Examples include light emitting diodes and solar cells. Some of the characteristics of InP are listed in Table 1. In common with many semiconductors, both the principal phonon energies and the energies of shallow impurities in InP correspond to photon energies for electromagnetic radiation in the range $\sim 1-10 \mathrm{THz}$. Thus $\mathrm{THz}$ radiation is an excellent probe of phonons, electrons and holes in this material.

Table 1: Some properties of InP.

\begin{tabular}{|c|c|c|c|c|c|c|c|}
\hline$\epsilon(0)$ & $\epsilon(\infty)$ & $\begin{array}{c}\omega_{T O}(4.2 \mathrm{~K}) \\
\mathrm{cm}^{-1} \\
{[2]}\end{array}$ & $\begin{array}{c}w_{\text {To }}(300 \mathrm{~K}) \\
\mathrm{cm}^{-1} \\
{[2]}\end{array}$ & $\begin{array}{c}\omega_{L O}(4.2 \mathrm{~K}) \\
\mathrm{cm}^{-1} \\
{[2]}\end{array}$ & $\begin{array}{c}\omega_{L O}(300 \mathrm{~K}) \\
\mathrm{cm}^{-1} \\
{[2]}\end{array}$ & $\begin{array}{c}m_{e}^{*} / m_{o} \\
{[3]}\end{array}$ & $m_{h}^{*} / m_{o}$ \\
\hline 12.61 & 9.61 & $308.2 \pm 0.3$ & $303.7 \pm 0.3$ & $349.5 \pm 0.3$ & $345.0 \pm 0.3$ & 0.077 & 0.64 \\
\hline
\end{tabular}

\section{B. Experimental}

Nominally undoped, donor-doped and acceptor-doped samples have been studjed. Details of the samples are given in Table 2. Reflectivity spectra were measured at room temperature using a Bomem DA3 rapid-scan spectrometer. A room-temperature DTGS detector was employed. A polished brass block served as a reflectance reference. Transmission measurements were made using the Bomem spectrometer and a slow-scan Polytec spectrometer. The samples were wedged appropriately to suppress interference fringes. Liquid helium was the coolant. Low temperatures were obtained by pumping on the helium bath.

Table 2: Samples used in this investigation.

\begin{tabular}{cccc}
\hline \hline Label & Dopant & Concentration $\left(\mathrm{cm}^{-3}\right)$ & Source \\
\hline A & None & & Sumitomo \\
B & None & $\leq 10^{16}$ & MTI Corporation \\
\hline C & Zinc & $4 \times 10^{16}$ & MCP \\
D & Zinc & $3.5 \times 10^{18}$ & MCP \\
E & Zinc & $(5-8) \times 10^{18}$ & CrysTec \\
F & Zinc & $(8-10) \times 10^{18}$ & Sumitomo \\
\hline G & Sulphur & $7 \times 10^{18}$ & Sumitomo \\
\hline \hline
\end{tabular}




\section{Reflection spectroscopy: phonon energies and carrier concentration}

The $\mathrm{THz}$ reflectivity spectra of undoped InP yield the TO and LO phonon energies as well as the dielectric constant and damping terms. In addition, the charge carrier concentration may be determined in doped samples by this purely optical method. Examples are given using doped and nominally undoped InP from a variety of sources.

The data is fitted assuming a damped harmonic oscillator dielectric function and a free carrier contribution:

$$
\epsilon(\omega)=\epsilon(\infty)+\frac{\omega_{i}^{2}(\epsilon(0)-\epsilon(\infty))}{\omega_{i}^{2}-\omega^{2}-i \gamma \omega}-\frac{\epsilon(\infty) \omega_{p}^{2}}{\left(\omega^{2}+i \gamma_{p} \omega\right)}=\epsilon_{1}(\omega)+i \epsilon_{2}(\omega)=(n+i \kappa)^{2}
$$

Here $\omega_{t}$ is the TO phonon frequency, $\gamma$ the phonon damping term, $\omega_{p}$ the plasma frequency and $\gamma_{p}$ the plasma damping term. From the refractive index $n$ and the extinction coefficient $\kappa$, the reflectivity may be calculated using

$$
R=\frac{(n-1)^{2}+\kappa^{2}}{(n+1)^{2}+\kappa^{2}}
$$

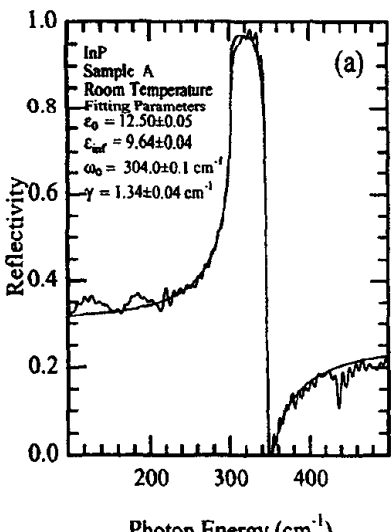

Photon Energy $\left(\mathrm{cm}^{-1}\right)$

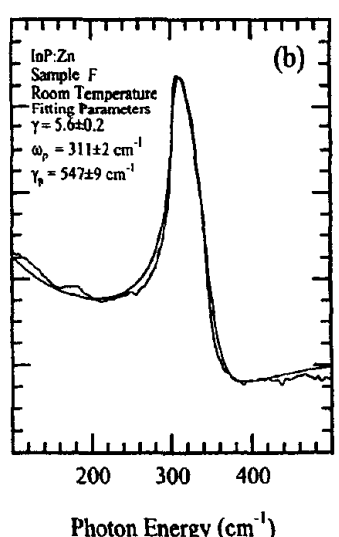

Photon Energy $\left(\mathrm{cm}^{-1}\right)$

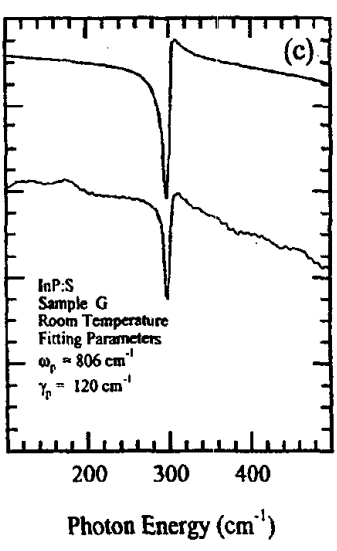

Fig. 1: Room-temperature reflectivity of samples $A, F$ and $G$.

Fig. 1 displays three distinctly different reflectivity spectra characteristic of undoped, lightly-doped and heavily-doped material, respectively. Fig. 1(a) shows the peak/dip characteristic of a slightly-damped harmonic oscillator, corresponding to undoped material. The materials parameters deduced by fitting Eqn. 1 to the experimental data are given in Fig. 1(a). Fig. 1(b) shows a reflectivity peak typical of a lightly-doped sample. The fitting parameters are also given. The carrier concentration, $N$, can be deduced from the plasma frequency, since $\omega_{p}^{2}=N e^{2} / \epsilon(\infty) \epsilon_{0} m^{*}$. Fig. 1(c) shows a sharp dip, characteristic of heavily-doped material. 


\section{Transmission spectroscopy: energy levels of shallow acceptors}

Shallow donor levels in InP have been studied for many years, most successfully by photoconductivity measurements made in magnetic fields using a far-infrared laser to excite the conductivity. Their energies and magnetic-field dependence are relatively well known. In comparison, the acceptor levels are less well known. Acceptor levels have been investigated by photoluminescence (PL) and considerable variation appears in the literature even for the $1 s_{3 / 2}\left(\Gamma_{8}^{+}\right)$ground state of common acceptors such as $\mathrm{Mg}$ and C. Recently, electronic Raman scattering was reported for $\mathrm{Zn}$ and $\mathrm{Cd}$ acceptors in InP $[4,5]$. This method is sensitive to transitions from the ground state to other evenparity states, such as $2 s_{3 / 2}\left(\Gamma_{8}^{+}\right)$. Transitions to odd-parity states are forbidden by the dipole selection rules. Absorption spectroscopy is complementary to this method, being sensitive to transitions to odd-parity final states.
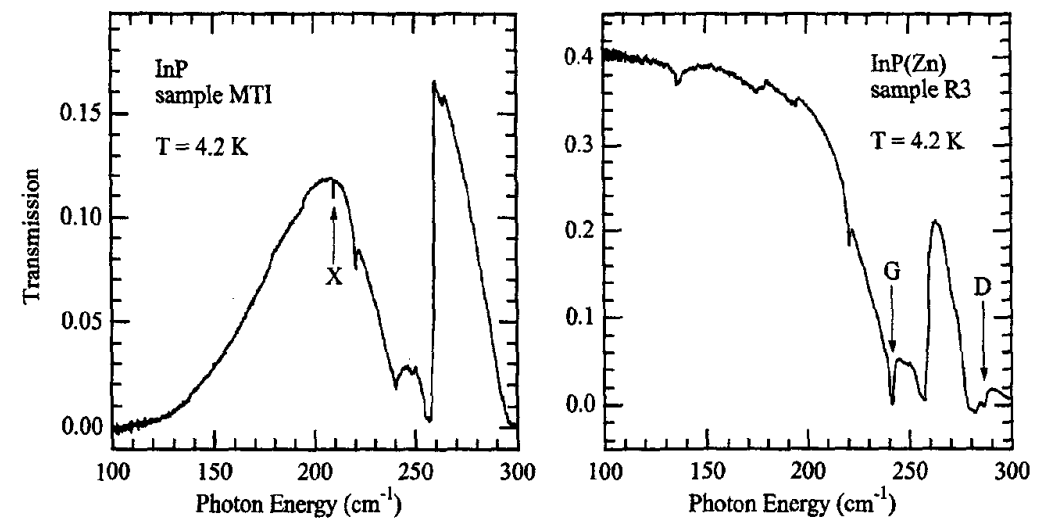

Fig. 2: Low-temperature transmission of samples B and C.

Transmission spectra over the range $100-300 \mathrm{~cm}^{-1}$ are given in Fig. 2 for samples $\mathrm{B}$ and C. Many of the features are in common (for example the sharp dip at $220 \mathrm{~cm}^{-1}$ and the edge at $260 \mathrm{~cm}^{-1}$ ) and may be attributed to lattice effects as comparison with earlier studies of the phonon spectra demonstrates [6]. Sample B exhibits a sharp absorption (marked "X") at $\sim 210 \mathrm{~cm}^{-1}$. Other measurements have indicated this feature (i) is very sharp; (ii) fades with temperature, disappearing by $60 \mathrm{~K}$; (iii) changes negligibly with magnetic field (to $6 \mathrm{~T}$ ). Spectra have been taken pumping on the helium space with no significant change in line width. The origin of this line is unknown. Sample $\mathrm{C}$ shows absorption at $241.5 \mathrm{~cm}^{-1}$ and $286.0 \mathrm{~cm}^{-1}$. These lines are identified as the Zn $G$ and $D$ lines. Data taken at higher temperatures confirm that they disappear due to thermal depopulation. The line positions from these spectra are given in Table 3 , along with previous measurements for transitions to other final states in InP. Ultrasonic treatment has been undertaken to reduce the linewidth but no narrowing is observed. To our knowledge, these are the first absorption spectra reported for acceptors in $\operatorname{InP}$. 
Table 3: Experimental energies (in $\mathrm{cm}^{-1}$ ) of transitions for acceptor impurities in InP.

\begin{tabular}{|c|c|c|c|c|c|c|}
\hline \multirow[t]{2}{*}{ Impurity } & & \multicolumn{3}{|c|}{ Zinc } & \multirow{3}{*}{$\frac{\text { Cadmium }}{[4]}$} & \multirow{3}{*}{$\begin{array}{c}\text { Carbon } \\
{[7]} \\
P[\end{array}$} \\
\hline & & This work & {$[4]$} & {$[7]$} & & \\
\hline Transition & Label & Absorption & Raman & PL & & \\
\hline $1 s_{3 / 2} \rightarrow 2 p_{3 / 2}$ & $G$ & 241.5 & 242.0 & 243 & 276.1 & 193 \\
\hline $1 s_{3 / 2} \rightarrow 2 s_{3 / 2}$ & $E$ & & 259.8 & 261 & 286.4 & 221 \\
\hline $1 s_{3 / 2} \rightarrow 2 p_{5 / 2}\left(\Gamma_{8}^{-}\right)$ & $D$ & 286.0 & 287.2 & 281 & 318.1 & 232 \\
\hline $1 s_{3 / 2} \rightarrow 2 p_{5 / 2}\left(\Gamma_{7}^{-}\right)$ & $\bar{C}$ & & 300.4 & 302 & 336.0 & 252 \\
\hline $1 s_{3 / 2} \rightarrow 3 s_{3 / 2}$ & 1 & & 318.7 & & & \\
\hline $1 s_{3 / 2} \rightarrow 4 s_{3 / 2}$ & 2 & & 330.6 & & & \\
\hline
\end{tabular}

\section{E. Conclusion}

The utility of $\mathrm{THz}$ radiation for the characterisation of $\mathrm{InP}$ has been illustrated. Reflection spectroscopy is a non-destructive, contactless, local probe which can be employed over a wide temperature range. It yields TO and LO phonon energies and carrier concentrations.

We report on the first absorption spectra of acceptors in InP. Our work on the $G$ and $D$ lines has now been extended to embrace Zeeman studies over a range of temperatures $(1.9-10.4 \mathrm{~K})$ and magnetic fields $(0-6 \mathrm{~T})$ for radiation polarised both parallel and perpendicular to the applied magnetic field. These investigations will be reported elsewhere.

\section{Acknowledgements}

This work is supported by the Australian Research Council and the University of Wollongong. We thank Professor C. Jagadish (Australian National University) and Dr. G. Irmer (Technische Universität Bergakademie Freiberg) for the provision of some of the samples used in this investigation.

\section{References}

[1] M. Hass and B. W. Henvis, J. Phys. Chem. Solids 23 (1962) 1099.

[2] A. Mooradian and G. B. Wright, Solid State Commun. 4 (1966) 431.

[3] K. W. Böer, "Survey of Semiconductor Physics" (Volume 2, Van Nostrand Reinhold, New York, 1992).

[4] G. Irmer, M. Wenzel and J. Monecke, phys. stat. sol. (b) 210 (1998) 347.

[5] M. Wenzel, G. Irmer and J. Monecke, Solid State Commun. 104 (1997) 371.

[6] E. S. Koteles and W. R. Datars, Solid State Commun. 19 (1976) 221.

[7] P. J. Dean, D. J. Robbins and S. G. Bishop, J. Phys. C: Solid State Phys. 12 (1979) 5567. 